Kai A. Konrad

Florian Morath

\title{
Bargaining with Incomplete Information: Evolutionary Stability in Finite Populations
}

\author{
Max Planck Institute for Tax Law and Public Finance \\ Working Paper 2014 - 16
}

September 2014

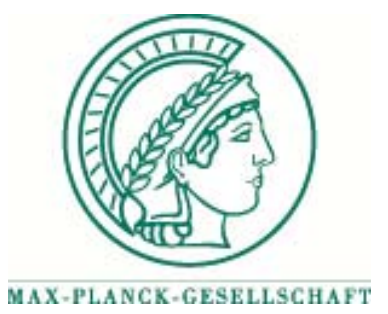

Max Planck Institute for

Tax Law and Public Finance

Department of Business and Tax Law

Department of Public Economics

http://www.tax.mpg.de 
Working papers of the Max Planck Institute for Tax Law and Public Finance Research Paper Series serve to disseminate the research results of work in progress prior to publication to encourage the exchange of ideas and academic debate. Inclusion of a paper in the Research Paper Series does not constitute publication and should not limit publication in any other venue. The preprints published by the Max Planck Institute for Tax Law and Public Finance represent the views of the respective author(s) and not of the Institute as a whole. Copyright remains with the author(s).

Max Planck Institute for Tax Law and Public Finance

Marstallplatz 1

D-80539 Munich

Tel: $\quad+498924246-0$

Fax: $\quad+498924246-501$

E-mail: ssrn@tax.mpg.de

http://www.tax.mpg.de 


\title{
Bargaining with incomplete information: Evolutionary stability in finite populations*
}

\author{
Kai A. Konrad $\quad$ Florian Morath ${ }^{\ddagger}$
}

August 29, 2014

\begin{abstract}
This paper considers evolutionarily stable strategies (ESS) in a take-it-orleave-it offer bargaining game with incomplete information. We find responders reject offers which yield a higher positive material payoff than their outside option. Proposers, in turn, make more attractive offers than in the perfect Bayesian equilibrium. Efficiency-enhancing trade may break down even when the responder has no private information. Overall, the probability of trade and ex post efficiency is lower in the ESS equilibrium than in the corresponding perfect Bayesian equilibrium. The results are observationally equivalent to behavioral explanations such as in-group favoritism and a preference for punishing selfish proposers but are driven by concerns about relative material payoff in finite populations.
\end{abstract}

Keywords: Evolutionary stability; Finite population; Take-it-or-leave-it offer bargaining; Asymmetric information

JEL Codes: C73; C78; D82

${ }^{*}$ Financial support from the German Research Foundation (DFG, grant no. SFB-TR-15) is gratefully acknowledged.

${ }^{\dagger}$ Max Planck Institute for Tax Law and Public Finance, Marstallplatz 1, D-80539 Munich, Germany; e-mail: kai.konrad@tax.mpg.de.

${ }_{\ddagger}^{\ddagger}$ Max Planck Institute for Tax Law and Public Finance, Marstallplatz 1, D-80539 Munich, Germany; e-mail: florian.morath@tax.mpg.de. 


\section{Introduction}

Bargaining under incomplete information has been studied extensively. One of the key insights was gained early on by Chatterjee and Samuelson (1983) and Myerson and Satterthwaite (1983): Bargaining under incomplete information may systematically fail to reach an efficient outcome. Deals that increase both sides' material payoff need not take place. We consider how the outcome of bargaining games with incomplete information is modified if players pursue evolutionarily stable strategies, based on the characterization of evolutionary stability in finite populations developed by Schaffer (1988) and by Nowak et al. (2004). ${ }^{1}$

We study the bargaining problem in a simple but generic framework in which the inefficiency caused by incomplete information is unveiled: ultimatum bargaining. A seller possesses a good. A buyer would like to buy the good. One of them makes a price offer to the other player. An exchange of the good takes place if the other player accepts the transaction price offered. The buyer and seller attribute material values to possession of the good. The buyer's material valuation of the good is common knowledge. The seller's material valuation is the seller's private information.

In the main part of the analysis we consider a price offer which is made by the uninformed buyer. The seller can either accept or reject. Applying the standard concept of perfect Bayesian equilibrium (PBE), the buyer as proposer faces a tradeoff. If he offers a high price, this may increase the likelihood of acceptance, but it also increases what the buyer has to pay should a trade take place. Depending on the players' actual valuation, the buyer's payoff-maximizing price offer can lead to failure of trade, even though the trade would be mutually beneficial. This is the basic and well-known inefficiency that emerges in the PBE among players who care only about their material payoff. Starting from there, we replace the equilibrium concept with evolutionary stability in finite populations. This brings in a different logic of decision making, which searches for strategies that maximize the players' fitness. With finite populations, the focus on evolutionary fitness adds an element of relative standing. We solve for evolutionarily stable strategies in this context and compare the outcome to the PBE.

We also consider bargaining if the informed player - the seller in our context

\footnotetext{
${ }^{1}$ Schaffer's (1988) concept of evolutionary stability in finite populations is a generalization of the seminal contributions of Maynard Smith and Price (1973) and Maynard Smith (1974) in the context of infinite populations.
} 
- makes an offer and the uninformed buyer accepts or rejects. In the context of evolutionarily stable strategies this case has some surprising features, and mutually beneficial trades in terms of material payoffs may not be realized. This contrasts with a corresponding PBE in this case which implements full efficiency.

The consequences of replacing the PBE concept by the concept of evolutionary stability are not straightforward. Evolutionary concepts highlight the importance of relative payoff, i.e., own material payoff relative to the material payoff of others. As Schaffer (1988) and a number of applications demonstrate, this may generally make players more "aggressive" than if they simply maximized their own material payoff. They may adopt a strategy that yields a lower own material payoff if this strategy reduces the average material payoff of the remainder of the population by even more. ${ }^{2}$ However, as has been shown by Eaton, Eswaran and Oxoby (2011) and Konrad and Morath (2012), evolutionarily stable strategies may also be described by in-group favoritism: behavior that resembles altruism. This is relevant when players interact in smaller subgroups of the population and their own performance is compared to that of the whole population. Players may behave more cooperatively in the specific bargaining interaction within the small set of players in a two-person subgroup, if the mutual benefit of this cooperative in-group behavior increases both players' payoffs relative to the material payoff of the rest of the population. The bargaining context here has a structure that provides opportunity for the emergence of such in-group favoritism: The population is grouped into small subgroups that consist of pairs of players, and the respective players in each pair interact as an in-group when bargaining with each other.

Our results show that evolutionarily stable strategies make agreement less likely, compared to the PBE with players who maximize their absolute material payoff. The responder behaves "tougher" than in the PBE. As a seller he rejects some offers for which the offered price exceeds his own material valuation of keeping the good. In this case he sacrifices his own material reward. The proposer behaves more generously and offers a higher price in the ESS than in the PBE. This may look like in-group favoritism, but from the perspective of the proposer it may be seen as an accommodating reaction to the tough behavior of the responder. And the accommodating

\footnotetext{
${ }^{2}$ Related to this, a literature based on a seminal paper by Mui (1995) highlights the role of envy. Evolutionarily stable strategies in small populations can actually provide an economic underpinning for envy, and the strength of such emotions may relate to group size and the type of interaction.
} 
behavior is not sufficient to overcome the primary, efficiency-reducing effect of tough responder behavior. Thus, the probability of trade and the expected material payoffs of players are lower in the equilibrium in evolutionarily stable strategies than in the PBE. This effect is strongest in small populations and becomes less pronounced if the population size is larger. ${ }^{3}$ We also show that a similar logic applies for evolutionarily stable strategies if the informed seller makes an ultimatum price offer to the uninformed buyer.

Related research has been pursued on evolutionarily stable strategies in the context of bargaining. Heifetz and Segev (2004) consider the strategic role of "toughness" in a bargaining context. This approach exploits the strategic commitment value of a toughness preference of the responder if the preference type is observed by the proposer. ${ }^{4}$ Their bargaining framework is related to ours, but the strategic benefit of being tough relies on type observability in their framework. A proposer who observes that his responding co-player is "tough" accommodates for the toughness and makes a more generous offer. Observed toughness has a strategic effect which benefits the tough player. In our framework, types are unobserved and therefore have no strategic commitment value. Huck and Oechssler (1999) consider a reduced form of the standard ultimatum offer game with perfect and complete information. They allow for a minimal set of possible proposer strategies: either offer a fair split or offer zero. ${ }^{5}$ Their evolutionary stability criterion is not identical but is closely related to the criterion developed by Schaffer (1988). Huck and Oechssler find that behavior by which

\footnotetext{
${ }^{3}$ We show that evolutionarily stable behavior converges toward Nash equilibrium behavior if the population size goes to infinity, a result which is also obtained in various contexts for the case of complete information. See Ania (2008) and Hehenkamp, Possajennikov and Guse (2010) for discussions on the equivalence of Nash equilibria and evolutionary stable equilibria in finite populations.

${ }^{4}$ For an evolutionary underpinning of different types of (other-regarding) preferences based on their strategic effects see also Sethi (1996), Bester and Güth (1998), Koçkesen, Ok and Sethi (2000), Dufwenberg and Güth (2000), Possajennikov (2000), Ok and Vega-Redondo (2001), Sethi and Somanathan (2001) and Dekel, Ely and Yilankaya (2007). For a recent contribution that addresses the evolutionary stability of preferences under incomplete information in an assortative matching game see Alger and Weibull (2013).

${ }^{5}$ See also Gale, Binmore and Samuelson (1995) and Nowak, Page and Sigmund (2000) for an evolutionary analysis of the ultimatum game, Binmore, Piccione and Samuelson (1998) for alternatingoffer bargaining and Ellingsen (1997) for the evolutionary stability of "obstinate" types in a Nash demand game. Abreu and Sethi (2003) consider a bargaining model with behavioral types that never concede to the opponent's demand, assuming type unobservability. For work on the evolutionary stability of trading mechanisms (instead of strategies and preferences, respectively) see, for instance, $\mathrm{Lu}$ and McAfee (1996).
} 
players reject zero offers is evolutionarily stable and can be generated as the subgame perfect equilibrium behavior of players with behavioral preferences. We widen the scope to the full continuous action space for take-it-or-leave-it bargaining; also, we depart from Huck and Oechssler (1999) by considering incomplete information. ${ }^{6}$ Our focus is on the well-known breakdown of trade in the PBE due to incomplete information and we ask whether evolutionarily stable behavior may widen or narrow the range in which trade takes place successfully.

We proceed as follows. The next section describes the framework and a criterion for evolutionary stability. Section 3 considers one-sided incomplete information and offers made by the uninformed player. Section 4 discusses the inverse case with offers made by the informed player. Section 5 concludes.

\section{The framework}

The setup. We study an evolutionary framework with a finite population of $n=2 \mathrm{~m}$ players in each generation. The state game consists of bilateral bargaining interactions within randomly formed subgroups of two players each. Within each two-player subgroup, nature randomly assigns the roles of seller and buyer. Each player has the same probability of being assigned the role of seller or buyer. ${ }^{7}$

The seller owns a resource which, if kept, gives him material value $v_{S}$. This material value $v_{S}$ is an independent draw from a uniform distribution on the unit interval $[0,1]$ and the value of $v_{S}$ is the seller's private information. The buyer attributes a material value $v_{B}$ to obtaining possession of the resource, which is commonly known. We study a benchmark case with $v_{B}=1$ in which trade is always efficient in terms of aggregate material payoff.

The two players interact in take-it-or-leave-it offer bargaining: One player makes a

\footnotetext{
${ }^{6}$ The indirect approach (in which evolution operates on objective functions) evokes difficult questions when it addresses incomplete information (see Konrad and Morath 2012 for a discussion and a first approach). Also, there is a well-known multiplicity of behavioral preferences that typically support the same strategy choice in the direct approach. Huck and Oechssler (1999) consider fairness or a preference for punishment. Bar-Gill (2006) considers optimism in bargaining problems. Other important candidates are in-group favoritism and out-group spite (Eaton, Eswaran and Oxoby 2011).

${ }^{7}$ To avoid an ex post asymmetry stemming from this assignment, we could allow each individual to be teamed up twice in each generation, once as a proposer and once as a responder. This would add complexity to the analysis. However, it is not essential for our results, as the equilibrium concept focuses on expected relative payoff.
} 
price offer and the other player accepts or rejects this price offer. In the main analysis we focus on the case in which the uninformed player (the buyer in our case) has the role of proposer and the informed player (the seller in our case) is the responder, as this is the scenario that is known to cause inefficiency in a standard perfect Bayesian game. ${ }^{8}$

In each matched pair of players the buyer proposes buying the resource for a payment $b \in[0, \infty)$. The seller as responder accepts or rejects this offer. If the offer is accepted, the buyer pays $b$ and receives the resource. This ends the game. The material payoffs are $v_{B}-b=1-b$ for the buyer and $b$ for the seller. If the offer is rejected, the seller keeps the resource and this ends the game. The material payoffs are zero for the buyer and $v_{S}$ for the seller in this case.

Evolutionary strategies consist of a vector $\left(b, \beta\left(v_{S}\right)\right)$ with two components. Here, $\beta$ is a function of own valuation of possession of the resource. The function

$$
\beta\left(v_{S}\right):[0,1] \rightarrow[0, \infty)
$$

describes a threshold strategy: A seller/responder with a valuation $v_{S}$ of keeping the good accepts an offer $b$ if and only if $b \geq \beta\left(v_{S}\right)$. Note that, if $\beta\left(v_{S}\right)>v_{S}$, the seller chooses a threshold for acceptance that is higher than his material valuation and, hence, sacrifices own material payoff by rejecting offers $b \in\left[v_{S}, \beta\left(v_{S}\right)\right]$. The opposite holds for $\beta\left(v_{S}\right)<v_{S}$. We restrict consideration to pure strategies and strictly increasing threshold functions $\beta .^{9}$ Let us also define the function $V_{S}(\cdot)$ as the inverse function of $\beta\left(v_{S}\right)$ where it exists. The function $V_{S}(b)$ determines the upper end of the range $v_{S} \in\left[0, V_{S}(b)\right]$ for which the seller/responder is willing to accept a given offer $b$ of the buyer.

Defining evolutionary stability. For a definition of evolutionarily stable strategies (ESS) and one-step mutations, suppose that $2 m-1$ players follow a given strategy $\sigma=(b, \beta)$ that determines a player's actions in the different roles as functions of their valuations. We distinguish between one-step proposer mutations, i.e., a

\footnotetext{
${ }^{8}$ In Section 5 we discuss a reversal of the roles of proposer and responder and assume that the informed seller makes a price offer and the uninformed buyer is the responder.

${ }^{9}$ Note that the assumption of well-behaved equilibrium strategies is important for the set of equilibria; see also the related discussion in Chatterjee and Samuelson (1983) on the corresponding case in which players maximize material payoff.
} 
buyer/proposer who makes an offer that deviates from $b$, and one-step seller/responder mutations, i.e., deviations from $\beta\left(v_{S}\right)$. We denote such deviations from the candidate evolutionarily stable strategy $\sigma$ by a superscript " $M$ ". Hence, a mutant strategy along the buyer dimension would be described by an offer $b^{M}$, and $\beta^{M}\left(v_{S}\right)$ would denote a possible mutant strategy in the seller dimension.

Denote by $\left(\sigma^{M}, \boldsymbol{\sigma}_{-M}\right)$ the strategy profile if one individual chooses $\sigma^{M}$ but all remaining individuals choose $\sigma$. Focusing on evolutionary fitness in finite populations within the concept of Schaffer (1988), we are interested in the players' ex ante expected material payoffs. To derive these, we first consider the timing of the resolution of uncertainty and of the players' moves in a given state game. Each of the $n=2 m$ players is programmed to follow a strategy $\left(b, \beta\left(v_{S}\right)\right)$, which defines the player's 'type.' We allow $\beta\left(v_{S}\right)$ to be a function of the player's own valuation of keeping the good, but we assume that co-players' specific types cannot be observed. This rules out that buyers/proposers or sellers/responders condition their programmed action on their respective co-player's type. ${ }^{10}$ This also makes sure that our findings are not driven by the 'strategic' value of players' types.

The evolutionary stability of a strategy is assessed on the basis of expected payoffs conditional on what is known in stage 0 : The distribution of strategies in the population is known, but matching has not taken place and the roles of proposer or responder have not been assigned. Also, only the distribution of the idiosyncratic valuation $v_{S}$ of the resource is known at stage 0 . For $v_{B}$, the valuation $v_{B}=1$ is common knowledge. We define by $E_{0} \Pi^{i}\left(\sigma^{i}, \boldsymbol{\sigma}_{-i}\right)$ the expected material payoff of player $i$ given the state of information in stage 0 . Note that players are symmetric at this stage. At stage 1 , players learn the group and the role $(S$ (eller)/responder or $B$ (uyer)/proposer) they are assigned to. We define by $E_{1} \Pi_{B}\left(\sigma^{i}, \boldsymbol{\sigma}_{-i}\right)$ and $E_{1} \Pi_{S}\left(\sigma^{i}, \boldsymbol{\sigma}_{-i}\right)$ the expected material payoffs of players at this stage 1 , conditional on their roles, but prior to learning

\footnotetext{
${ }^{10}$ Evolutionary game theory has considered the role of "greenbeards," the phenomenon that individuals condition their behavior on the (observable) type of their co-player. This type of conditional play is the basis for much of the results that allow for other-regarding behavior in the equilibrium. This type of phenomenon is not the basis for our results. Note further that the proposer's choice is also not conditional on the responder type. The offer $b$ chosen by a player in the proposer role is independent of the responder type of the player who responds. This, in turn, eliminates "strategic" incentives that have also been prominent in explaining other-regarding preferences and deviations from Nash behavior in the evolutionary equilibrium, e.g., in Robson (1990), Güth and Yaari (1992), Banerjee and Weibull (1993), and Bester and Güth (1998). See also Ely and Yilankaya (2001) and Dekel, Ely and Yilankaya (2007) on observable versus non-observable preference types and the relation to Nash behavior.
} 


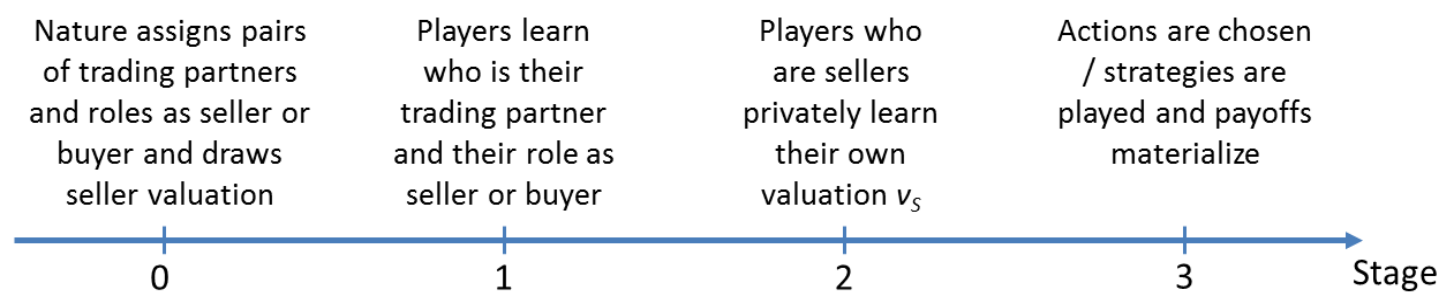

Figure 1: Time structure of the state game

about $v_{S}$. At stage 2, a player in role $S$ learns his $v_{S}$; then, the players take actions according to their strategies $\left(b, \beta\left(v_{S}\right)\right)$. Figure 1 summarizes the time structure.

We can now use Schaffer (1988) to define:

Definition 1 The strategy $\sigma$ is an evolutionarily stable strategy ("ESS") if there is no one-step mutation $\sigma^{M}$ from $\sigma$ such that

$$
E_{0} \Pi^{M}\left(\sigma^{M}, \boldsymbol{\sigma}_{-M}\right)-E_{0} \Pi^{-M}\left(\sigma^{M}, \boldsymbol{\sigma}_{-M}\right)>0 .
$$

As has been discussed in the theory of evolutionary dynamics, the composition of finite populations develops stochastically. The strong results obtained for a continuum of individuals do not apply in finite populations. The 'fixation probability' for any type, i.e., the probability that a single mutant of a given type manages to take over the whole population, is usually smaller than 1 . Rather, evolutionary stability of a particular type typically does not imply that a population is completely immune to invasion and cannot be taken over by a mutant type. However, the criterion (1) appears as the central condition in Schaffer (1988) and is also a necessary condition in a stochastic dynamic theory (see Nowak et al. 2004, Taylor et al. 2004, or Nowak 2006, condition (7.16)).

The criterion has a nice intuition that has been emphasized by Schaffer (1988). Suppose that a mutation appears in a given generation, which otherwise consists of $n-1$ players of a homogeneous 'old' type. All $n$ players now interact, pairwise, as in our context. If this mutant has a higher expected material payoff than the average expected material payoff of all other players in this generation, it does not warrant final success, but it is a good start for the mutant type. The criterion rules out that mutations with this kind of a good start exist. In order to have such a good start, a 
mutant's material payoff does not need to be higher than the average material payoff that emerged for 'old' type players if they interact only with homogeneous players of the 'old' type. The mutant can still do well even if the mutant's material payoff is lower than this material payoff $\Pi(\sigma, \boldsymbol{\sigma})$ which 'old' types achieve in the absence of any mutant. This can happen if the average material payoff of the 'old' types is sufficiently reduced if the population is invaded by a mutant. What matters is the comparison of the mutant's material payoff and the material payoff of the 'old' types in a population with a mutant.

\section{Equilibrium in ESS}

The following proposition characterizes the evolutionarily stable strategies of players in their roles of buyer/proposer and seller/responder:

Proposition 1 An equilibrium in evolutionarily stable strategies is characterized by

$$
b=\frac{1}{2} \frac{4 m^{2}-2 m-1}{4 m^{2}-2 m-m}
$$

and

$$
\beta\left(v_{S}\right)=v_{S}+\frac{1-v_{S}}{2 m} .
$$

Hence, sellers/responders accept an offer $b$ if and only if $v_{S} \in\left[0, V_{S}(b)\right]$, where

$$
V_{S}(b)=\frac{2 b m-1}{2 m-1}
$$

Proof. First, we search for the mutant seller/responder strategy that maximizes the mutant's relative material payoff in (1) for a given offer $b$. Second, we search for the mutant buyer/proposer strategy that maximizes (1), given that all players in the role of seller/responder use the strategy characterized by (4).

Step 1: seller/responder strategy mutation: Consider a population with $2 m-1$ ESS players and one mutant. This mutant chooses the ESS offer $b$ if the mutant is assigned the role of buyer/proposer. ${ }^{11}$ He chooses $V_{S}^{M}(b) \neq V_{S}(b)$ if he is in the seller/responder role. Thus, if the mutant acts as the buyer/proposer, the mutant

\footnotetext{
${ }^{11}$ Note that here and in the following we omit the superscript "ESS" at all ESS choices but only indicate mutations from ESS by the superscript "M".
} 
chooses $b$ similar to an ESS player. The mutant's expected material payoff in the role of buyer is

$$
\int_{0}^{V_{S}(b)}\left(v_{B}-b\right) d v_{S}=V_{S}(b)(1-b) .
$$

If the mutant acts as a seller/responder, he chooses the mutant threshold level $V_{S}^{M}$. As the mutant interacts with an ESS buyer/proposer, the mutant's expected payoff in the role of the seller is

$$
\int_{0}^{V_{S}^{M}(b)} b d v_{S}+\int_{V_{S}^{M}(b)}^{1} v_{S} d v_{S}=V_{S}^{M}(b) b+\frac{1}{2}\left(1-\left(V_{S}^{M}(b)\right)^{2}\right) .
$$

The mutant has the buyer role and the seller role with probability $1 / 2$. Accordingly,

$$
E_{0} \Pi^{M}=\frac{1}{2} V_{S}(b)(1-b)+\frac{1}{2}\left[V_{S}^{M}(b) b+\frac{1}{2}\left(1-\left(V_{S}^{M}(b)\right)^{2}\right)\right] .
$$

The mutant behavior also influences the average expected material payoff of a non-mutant. A non-mutant may have the role of buyer/proposer or seller/responder, and may interact with a non-mutant or a mutant. The following matrix summarizes possible matching combinations, their frequencies and payoffs:

$$
\begin{array}{ccc}
\multicolumn{1}{c}{\begin{array}{c}
\text { matched } \\
\text { ESS-player is } \backslash \text { co-player is }
\end{array}} & \text { non-mutant } & \text { mutant } \\
\text { buyer/proposer } & V_{S}(b)(1-b) & V_{S}^{M}(b)(1-b) \\
& \frac{2 m-2}{2 m-1} & \frac{1}{2 m-1} \\
\text { seller/responder } & V_{S}(b) b+\frac{1}{2}\left(1-\left(V_{S}(b)\right)^{2}\right) & V_{S}(b) b+\frac{1}{2}\left(1-\left(V_{S}(b)\right)^{2}\right) \\
& \frac{2 m-2}{2 m-1} & \frac{1}{2 m-1}
\end{array}
$$

For instance, the upper-left payoff is the payoff of a non-mutant player who is assigned the role of buyer/proposer and meets another non-mutant player who must then be a seller/responder. There are $2 m-1$ 'other' players, and $2 m-2$ of them are non-mutants. Note that the payoff entries for the left and right lower cells are identical: If the mutant player is a mutation along the seller-responder dimension, then in the role of buyer/proposer the mutant acts like a non-mutant. For a seller/responder non-mutant it therefore does not matter if the co-player is a mutant or not. Taking into account that any given non-mutant has a probability of $1 / 2$ of becoming a 
buyer/proposer or seller/responder, the expected payoff of a non-mutant at stage 0 is

$$
\begin{aligned}
E_{0} \Pi^{-M}= & \frac{1}{2}\left[\frac{1}{2 m-1} V_{S}^{M}(b)(1-b)+\frac{2 m-2}{2 m-1} V_{S}(b)(1-b)\right] \\
& +\frac{1}{2}\left[V_{S}(b) b+\frac{1}{2}\left(1-\left(V_{S}(b)\right)^{2}\right)\right] .
\end{aligned}
$$

Maximizing the difference $E_{0} \Pi^{M}-E_{0} \Pi^{-M}$ with respect to $V_{S}^{M}$ yields the first order condition

$$
\frac{1}{2}\left(b-V_{S}^{M}\right)-\frac{1}{2} \frac{1}{2 m-1}(1-b)=0
$$

which is solved for $V_{S}^{M}=V_{S}$ as in (4). The function $\beta\left(v_{S}\right)$ in (3) is obtained by solving (4) for $b$.

Step 2: buyer/proposer strategy mutation: Now consider a population with $2 m-1$ ESS players and one mutant who chooses the ESS value $V_{S}(b)$ if he is assigned to the role of seller/responder but deviates from the ESS strategy to $b^{M} \neq b$ if he is assigned the role of buyer/proposer. Analogous to the reasoning in step 1, we get the material payoff of the buyer/proposer mutant as

$$
E_{0} \Pi^{M}=\frac{1}{2} V_{S}\left(b^{M}\right)\left(1-b^{M}\right)+\frac{1}{2}\left[V_{S}(b) b+\frac{1}{2}\left(1-\left(V_{S}(b)\right)^{2}\right)\right] .
$$

Moreover, the average expected material payoff of a non-mutant at stage 0 becomes

$$
\begin{aligned}
& E_{0} \Pi^{-M}= \frac{1}{2} V_{S}(b)(1-b) \\
&+\frac{1}{2}\left[\frac{1}{2 m-1}\left(V_{S}\left(b^{M}\right) b^{M}+\frac{1}{2}\left(1-\left(V_{S}\left(b^{M}\right)\right)^{2}\right)\right)\right. \\
&\left.\quad+\frac{2 m-2}{2 m-1}\left(V_{S}(b) b+\frac{1}{2}\left(1-\left(V_{S}(b)\right)^{2}\right)\right)\right]
\end{aligned}
$$

Maximizing the difference $E_{0} \Pi^{M}-E_{0} \Pi^{-M}$ with respect to $b^{M}$ yields the first order condition

$$
\begin{aligned}
\frac{1}{2}\left[V_{S}^{\prime}\left(b^{M}\right)\left(1-b^{M}\right)\right. & \left.-V_{S}\left(b^{M}\right)\right] \\
& -\frac{1}{2} \frac{1}{2 m-1}\left[V_{S}^{\prime}\left(b^{M}\right) b^{M}+V_{S}\left(b^{M}\right)-V_{S}\left(b^{M}\right) V_{S}^{\prime}\left(b^{M}\right)\right]=0 .
\end{aligned}
$$

With (4) and

$$
V_{S}^{\prime}\left(b^{M}\right)=\frac{2 m}{2 m-1},
$$


the solution to the first order condition is $b^{M}=b$ as in (2).

Finally, note that $V_{S}(b)<0$ for all $b<1 /(2 m)$; hence, all offers lower than $1 /(2 m)$ are rejected with probability 1 . If $m=1$, this implies that the difference $E_{0} \Pi^{M}-E_{0} \Pi^{-M}$ is independent of $b^{M}$ for all $b^{M} \in[0,1 / 2]$. But by (2), $E_{0} \Pi^{M}-E_{0} \Pi^{-M}$ is maximized for $b^{M}=1 / 2$. In other words, if $m=1$, there is a continuum of payoffequivalent equilibria in which $b \in[0,1 / 2]$ and $V_{S}$ is as in (4). Intuitively, if $m=1$, a player who is assigned the role of buyer/proposer cannot do better than offering $b=1 / 2$; but since this offer is rejected with probability 1 , he can also offer less without affecting his or any other player's material payoff. Higher offers $b>1 / 2$, however, strictly reduce a player's fitness since they leave a large surplus for sellers/responders with a low material valuation $v_{S}$.

Before we proceed we discuss the properties of the ESS in Proposition 1. Note that $\beta\left(v_{S}\right)>v_{S}$ for all $v_{S}<1$. This means that some equilibrium offers are rejected even if the responder's valuation $v_{S}$ is strictly lower than these offers $b$. This corresponds to a general insight obtained in the context of evolutionary stability in small populations: The concern for relative material payoff makes spiteful strategies evolutionarily stable. To illustrate, consider a seller/responder who considers to accept an offer $b$ if $b=v_{S}+\varepsilon$ for small but positive $\varepsilon$. This seller gains a very small material payoff compared to not selling the resource. The buyer/proposer who makes such an offer receives a potentially much higher rent if the seller accepts such an offer. The buyer's rent is $1-b$ in case of acceptance, and zero otherwise. The harm imposed on such a buyer/proposer by rejecting outweighs the own sacrifice from rejecting.

The threshold function $\beta\left(v_{S}\right)$ for acceptance in (3) also shows that toughness is a function of the difference between the buyer/proposer's material payoff, which is equal to 1 , and the seller/responder's material payoff $v_{S}$ in case of acceptance. The divergence between the threshold $\beta\left(v_{S}\right)$ and $v_{S}$ is maximal for very small values of $v_{S}$. For $v_{S} \rightarrow 1$, the responder's threshold converges toward his own valuation of keeping the resource that could be traded.

Note further that $\beta\left(v_{S}\right) \rightarrow v_{S}$ if $m \rightarrow \infty$; hence, all offers with $b \geq v_{S}$ are accepted if the size of the population is sufficiently large. This outcome is similar to other applications of the concept of evolutionary stability in finite populations. The negative impact on the material payoff of the buyer which the seller has by rejecting the offer is less important if there is a large population with many players who are not directly affected by the particular choice of a given buyer/proposer or a given 
seller/responder.

The buyer/proposer offer in (2) is less straightforwardly in line with the general intuition for evolutionary stability in small groups. The buyer's choice can be seen as an accommodating reaction to the reluctance of the seller/responder to accept low price offers. As the seller requires a higher own material payoff and does not accept otherwise, the buyer offers to pay a higher price $b$. But accommodating the seller's tough reservation price only partially explains the buyer's offer behavior. The offer in (2) is also shaped by concerns for relative standing: The offer is strictly lower than the offer which would maximize the buyer/proposer's absolute material payoff for a given ESS of the seller/responder as in (4). As is straightforward to confirm, the best response to (4) that maximizes own material payoff would be

$$
\tilde{b}=\frac{1}{2}+\frac{1}{4 m}>b .
$$

The buyer/proposer does not want to offer as much as $\tilde{b}$ since such a high offer benefits the seller and thereby reduces the buyer's evolutionary fitness.

Finally, as for the seller/responder, the evolutionary effect for the buyer/proposer offer vanishes if the population size increases, which is also a consequence of the change in the responder strategy: $\lim _{m \rightarrow \infty} b=1 / 2$ (see below).

Comparison with standard equilibrium concepts. The take-it-or-leave-it offer bargaining game has been studied extensively and its solution has been used in many contexts to describe bargaining with incomplete information. We may contrast the equilibrium in ESS with Bayesian Nash equilibrium and with perfect Bayesian equilibrium (PBE) for rational players who aim at maximizing their own material payoff. The simultaneous choices of $b$ by the buyer/proposer and of the acceptance rule by the seller/responder as for Bayesian Nash equilibrium may correspond to the consideration of one-step deviations in the analysis of ESS. However, we concentrate on perfect Bayesian equilibrium, as the set of Bayesian Nash equilibrium is large and does not address the sequential nature of decision making. ${ }^{12}$ For the parametric case

\footnotetext{
${ }^{12}$ The set of Bayesian Nash equilibria is rather large. For instance, the seller/responder may choose to reject any price offer $b \neq b_{0}$ for some $b_{0}$, and to accept $b_{0}$ if and only if $v_{S} \leq b_{0}$. The buyer/proposer's optimal reply to this strategy is $b_{0}$ for any $b_{0} \leq 1$. In turn, the fact that the buyer always proposes $b=b_{0}$ makes rejecting all prices $b \neq b_{0}$ a best reply for the seller. Most of these equilibria do not survive reasonable refinements or sequential rationality. This is why we focus on the perfect Bayesian equilibrium.
} 
which we consider here, sequential rationality requires that the offer is accepted by the seller/responder if and only if $v_{S} \leq b=V_{S}^{N}(b)$. Here and below, the superscript " $N$ " refers to variables relating to the PBE, to distinguish the PBE values from the equilibrium values of ESS that have no superscript (or a superscript "ESS", where this may help for a distinction). Given the belief about the seller's reservation price, ${ }^{13}$ the PBE offer made by the proposer is $b^{N}=1 / 2$.

Comparing ESS and this PBE, we first note that

$$
\lim _{m \rightarrow \infty} b^{E S S}=b^{N} \text { and } \lim _{m \rightarrow \infty} V_{S}^{E S S}(b)=b=V_{S}^{N}(b) .
$$

(For a matter of comparison with the PBE values, we add a superscript "ESS" to the values of $b$ and $V_{S}$ that describe the ESS as characterized in Proposition 1.) Figure 2 compares the PBE outcome with the evolutionarily stable equilibrium, for different population sizes. The figure illustrates the divergence of the ESS offer $b^{E S S}$ and the critical ESS valuation $V_{S}^{E S S}\left(b^{E S S}\right)$, which determines acceptance of the ESS offer. The figure offers two insights.

First, the vertical distance between the two curves $b^{E S S}$ and $V_{S}^{E S S}$ represents the minimum rent which the responder requires for himself to be willing to accept the offer. This minimum rent is zero in the PBE since $b^{N}=V_{S}^{N}=1 / 2$ in this case. But acceptance of an offer also grants a rent to the proposer, and this matters for evolutionary stability since a positive rent of the proposer reduces the evolutionary fitness of the responder. Therefore, the ESS strategy $V_{S}$ requires a strictly positive rent in order to accept an offer, and this minimum rent is decreasing in the number of players.

Second, the buyer's offer interacts with the ESS strategy of the seller/responder. For $m=1$, the ESS offer is equal to $1 / 2$ and is thus equal to the offer in the PBE, but for any larger population size $m$, the equilibrium in ESS is characterized by an offer that is higher than $1 / 2 .{ }^{14}$ The relationship between the ESS offer $b$ and $m$ is non-monotonic; the ESS offer $b$ is increasing for $m<2$, has a maximum at $m=2$

\footnotetext{
${ }^{13}$ Note that there is some possible confusion about the meaning of 'type' here. The seller's reservation price is what is referred to as his 'type' in the context of standard concepts of Bayesian equilibrium, whereas in the context of evolutionary equilibrium, a player's type is identified with the player's actual action.

${ }^{14}$ Note that, if $m=1$, then $V_{S}(b)=0$ and $b$ is rejected with probability one. In fact, if $m=1$, then the buyer/proposer is indifferent between all $b \in[0,1 / 2]$; see the proof of Proposition 1 for details.
} 


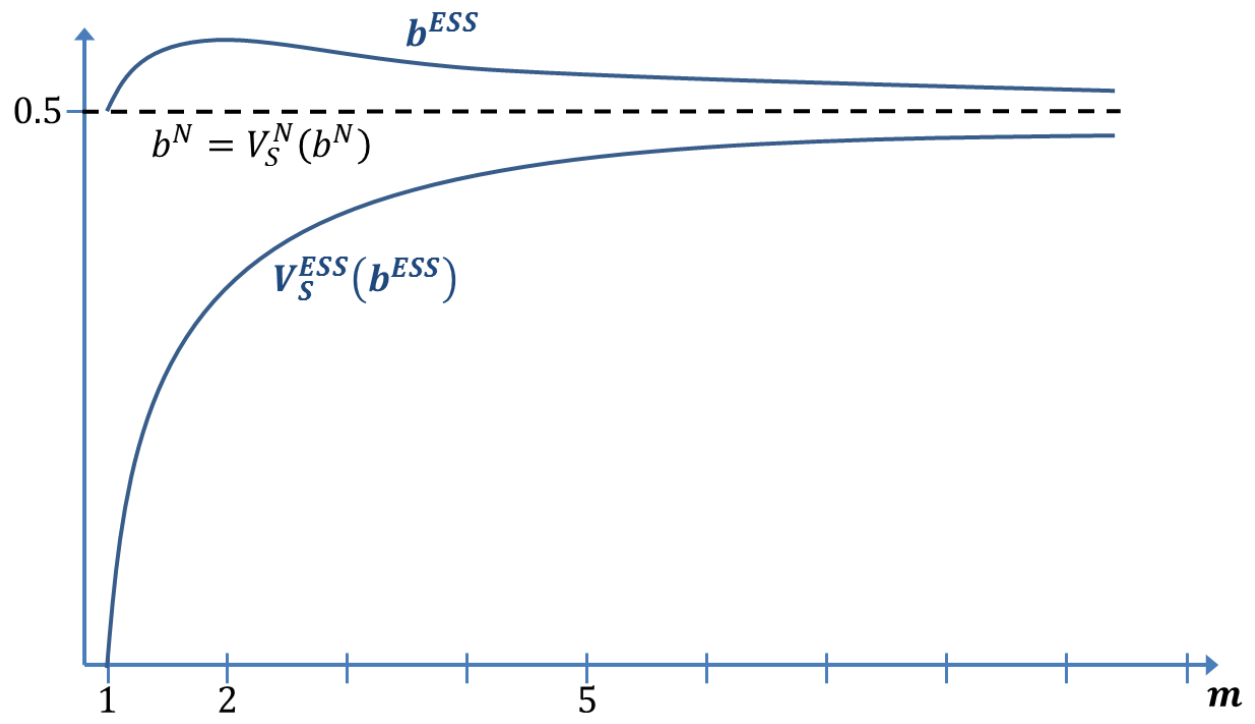

Figure 2: Comparison of the evolutionarily stable equilibrium and the perfect Bayesian equilibrium.

and is then decreasing in $m$, converging to $1 / 2$ for very large $m$. Intuitively, one main force that drives $b$ away from $1 / 2$ is the strong deviation of the seller/responder from the $\mathrm{PBE}$ value. In the limit case of $m=1$, even sellers with a very low $v_{S}$ require a high offer in order to accept; but it is evolutionary stable for the buyer not to offer such high amounts. If the population size increases, the seller/responder claims a lower surplus which makes it "cheaper" for the buyer/proposer to ensure a given probability of trade.

Probability of trade and ex-post efficiency. Now we can analyze the efficiency properties of the bargaining outcome in ESS and compare it with the PBE.

Proposition 2 Let $\omega$ be the probability of trade for ESS and $W$ be the expected total material payoff in the equilibrium in ESS for a single pair of players. It holds that

$$
\omega=\frac{1}{2}-\frac{1}{2} \frac{1}{4 m-3}
$$

and

$$
W=\frac{1}{2}-\frac{1}{2} \frac{(2 m-2)^{2}}{(4 m-3)^{2}}+\frac{2 m-2}{4 m-3}
$$


Both $\omega$ and $W$ are increasing in $m$ with $\lim _{m \rightarrow \infty} \omega=\frac{1}{2}=\omega^{N}$ and $\lim _{m \rightarrow \infty} W=\frac{7}{8}=$ $W^{N}$.

For the proof of Proposition 2 see the appendix. As is well-known from Schaffer (1988) and the applications of his definition in a number of contexts, in finite populations, it is not the absolute material payoff but a type's increase in relative material payoff that matters. In the bargaining context, this makes the sellers/responders "tough," that is, more reluctant to accept offers. The evolutionarily stable buyer/proposer strategy accommodates the seller/responder behavior, although relative payoff concerns prevent the buyer/proposer from making "too high" offers. Overall, for the probability of trade and for the total expected material payoff, the accommodating effect of the buyer/proposer is weaker than the increased toughness of the seller/responder. This widens the interval of material valuations for which trade does not take place, compared to the outcome in the PBE. In other words, the probability of trade $\omega$ and the aggregate resource rent $W$ are strictly lower for ESS than in the $\mathrm{PBE}$, but converge to the PBE values when $m$ becomes very large.

The analysis in this section focused on offers made by the uninformed buyer, giving all bargaining power to the uninformed player. This is the more relevant case for an efficiency comparison between ESS and PBE because the case of uninformed proposers and privately informed responders is the standard case known to cause inefficient failure to trade also in the PBE. However, it is an extreme case. The complementary case allocates all bargaining power to the informed player, which is the seller in our setup. We now turn to this problem.

\section{Informed proposers}

Suppose that, unlike in the previous sections, the informed seller is the player who proposes a price for the buyer to pay, and the uninformed buyer decides whether or not to accept it. Apart from this relocation of bargaining power, we keep all other building blocks of the formal framework unchanged. The seller has a valuation $v_{S} \in[0,1]$ which is drawn from a uniform distribution. The seller knows his own valuation, the buyer knows the distribution. The buyer has an exogenously given valuation $v_{B}=1$. This valuation is common knowledge. 
Evolutionarily stable strategies. We consider the following set of possible evolutionary strategies. The seller/proposer sets a price $p$, which may depend on the seller's true material valuation $v_{S}$ of keeping the good. Accordingly, strategies of the seller/proposer are defined as functions

$$
p\left(v_{S}\right):[0,1] \rightarrow[0,1]
$$

The strategies of the buyer/responder are defined by threshold values $\pi \in[0, \infty) .{ }^{15}$ The threshold $\pi$ is defined such that the buyer/responder accepts all prices for which $p \leq \pi$ and rejects all prices for which $p>\pi$. An evolutionary strategy $\left(p\left(v_{S}\right), \pi\right)$ consists of a seller/proposer strategy and a buyer/responder strategy. This leads to the following characterization of the equilibrium in evolutionarily stable strategies:

Proposition 3 There is a continuum of evolutionarily stable strategies $\left(p\left(v_{S}\right), \pi\right)$ such that a buyer/responder accepts the offer price $p$ if $p \leq \pi$ and rejects the offer otherwise and a seller/proposer offers to sell for $p=\pi$ if $v_{S} \leq P_{S}$ and offers to sell for $p=1$ if $v_{S}>P_{S}$, where $P_{S}$ is defined as

$$
P_{S}=\frac{2 m}{2 m-1} \pi-\frac{1}{2 m-1}
$$

and where

$$
\pi \leq \frac{8 m^{2}-8 m+1}{8 m^{2}-6 m}
$$

A proof of Proposition 3 is in the appendix. A few properties of evolutionarily stable strategies in Proposition 3 are intuitively straightforward. The buyer/responder does not know the true valuation $v_{S}$ of the seller/proposer. The buyer must choose whether to accept the price on the basis of the offer price $p$ only. The evolutionarily stable acceptance strategy by ESS buyers/responders is characterized by a threshold price $\pi$ : Buyers buy if the price offered does not exceed this threshold price. In any of the equilibria in ESS, the threshold price $\pi$ is strictly smaller than the buyer's material valuation $v_{B}=1$ (compare $\left.(8)\right)$.

The buyer's acceptance behavior, in turn, affects the considerations for the seller. Taking into account that the buyer/responder has a threshold strategy $\pi$, the optimal

\footnotetext{
${ }^{15}$ We could allow for an acceptance strategy of the buyer/responder that is non-monotonic in $p$. This may potentially lead to further ESS, but this does not justify the increase in complexity of the analysis caused by the possibility of arbitrary, non-monotonic acceptance strategies.
} 
offer strategy of the seller/proposer can make use of two possible offer prices only: $p \in\{\pi, 1\}$. Price offers higher than $\pi$ all lead to rejection (and are all equally profitable), and price offers $p<\pi$ would be accepted, but are inferior to offers of $p=\pi$.

Whether a seller/proposer offers to sell for $p=\pi<1$ (in which case he can sell with probability 1) or for $p=1$ (essentially deciding not to trade) depends on the benefit from trade for himself and for the buyer/responder. But from an evolutionary perspective, trade is worthwhile for the seller/proposer only if the own benefit from trade is sufficiently high in comparison to the benefit from trade at $p=\pi$ for the buyer/responder. Since the buyer's material benefit is independent of $v_{S}$ but the seller's material benefit from trade is lower the higher $v_{S}$, the comparison of costs and benefits explains the threshold for the seller's valuation in (7): From an evolutionary point of view, it is beneficial for a seller/proposer to sell for a price $p=\pi$ only if the own valuation of no trade is sufficiently small: $v_{S} \leq P_{S}$. Moreover, it is intuitive that the critical threshold $P_{S}$ for the seller's valuation is an increasing function of the possible transaction price $p=\pi$ : A higher transaction price for which the seller/proposer can sell the resource increases the seller's own material advantage from selling compared to keeping the good, and it lowers the buyer's material benefit from this purchase.

The ESS value of $P_{S}$ depends on $\pi$; in fact, for a given responder threshold $\pi$, the seller's offer function is uniquely determined. But the value of $\pi$ also depends on the ESS value of $P_{S}$. This is the case because when $\pi$ is determined the buyer/responder solves a similar trade-off as the seller/proposer as to whether to trade or not: For any price $p<1$, trade offers a material reward to the buyer/responder. But trade also offers a material reward to the seller if $p>v_{S}$, and this is bad for the buyer/responder from an evolutionary point of view. Closer inspection of the proof of Proposition 3 shows why there is a whole range of combinations $\left(P_{S}, \pi\right)$ that characterize evolutionarily stable strategies.

If $\pi$ is large, the material reward for the seller is large, and the material reward for the buyer is low. Hence, there is an upper bound on the evolutionarily stable $\pi$; for higher values of $\pi$, mutations in the role of the buyer which lead to the breakdown of trade would be evolutionarily advantageous. This explains the upper bound on $\pi$ in (8). 


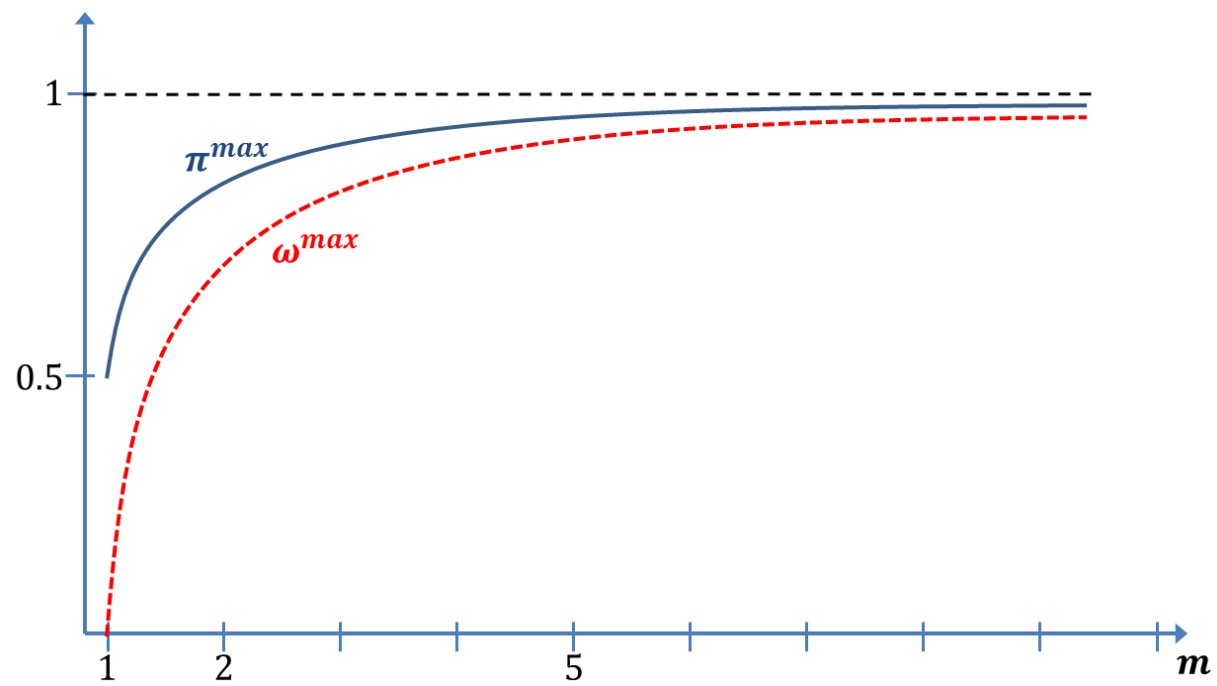

Figure 3: The set of evolutionarily stable equilibria in case of informed proposers.

The set of evolutionarily stable strategies is a function of the size of the population $n=2 m$, as illustrated in Figure 3. The solid function $\pi^{\max }(m)$ in Figure 3 represents condition (8); all $\left(p\left(v_{S}\right), \pi\right)$ with $0 \leq \pi \leq \pi^{\max }$ and the corresponding threshold $P_{S}$ for the seller's valuation as in (7) are evolutionarily stable. Since $v_{S}$ is a draw from the uniform distribution on the unit interval, the threshold value $P_{S}$ is also equal to the probability of trade. The dashed function $\omega^{\max }(m)$ in Figure 3 maps the highest achievable probability of trade for ESS (equation (7) evaluated at the upper bound on $\pi)$,

$$
\omega^{\max }=\frac{4(m-1)}{4 m-3}
$$

The upper bound $\omega^{\max }$ on the probability of trade is equal to zero for $m=1$ and converges to one if $m \rightarrow \infty$. Evolutionary considerations of the material payoff relative to the payoff of the matched partner are strongest in small populations. However, if there are many other players to compare with, then it is optimal for players to disregard the co-player's payoff and maximize absolute own material payoff. In the latter case, trade with a probability close to 1 can be achieved. The vertical difference between the functions $\pi^{\max }$ and $\omega^{\max }$ also illustrates the fact that $P_{S}<\pi$ : There is a range of valuations $v_{S} \in\left(P_{S}, \pi\right)$ for which trade would yield a material benefit to the seller but for which the seller decides not to trade. 
Comparison with the PBE among material payoff maximizing players. In close analogy to the ESS, there is a set of Bayesian Nash equilibria ${ }^{16}$ and a perfect Bayesian equilibrium. Sequential rationality requires that the buyer/responder accepts all price offers for prices smaller than or equal to 1 and rejects all prices higher than 1 . This leads to the PBE characterized by an offer $p^{N}=1$ and a choice by the buyer/responder to accept all price offers smaller than or equal to 1 . Trade always takes place and is efficient in this PBE, given our assumptions about $v_{S} \in[0,1]$ and $v_{B}=1$.

For finite $m$, however, the set of ESS does not include the efficient case of $\left(P_{S}, \pi\right)=$ $(1,1)$. Intuitively, the buyer/responder has an own material payoff of zero if trade takes place at $(1,1)$-just as much as if no trade takes place. However, the buyer can reduce the seller's material payoff (who would get $\pi=1$ in case of trade) by choosing not to trade: In the "no trade" case the expected material payoff of the seller is $E\left(v_{S}\right)=1 / 2<1$.

\section{Conclusions}

The evolutionarily stable equilibrium of the take-it-or-leave-it offer bargaining game with one-sided incomplete information is characterized by a low probability of trade, compared to perfect Bayesian equilibrium. The breakdown of trade is a well-known possible outcome in the perfect Bayesian equilibrium of take-it-or-leave-it offer bargaining if the uninformed party makes an ultimatum offer to a privately informed responder. For evolutionarily stable strategies, the parameter range in which trade takes place is even more limited than for perfect Bayesian equilibrium if the uninformed player makes the offer. This result carries over to the case where the informed player makes the ultimatum offer. The main reason behind the low-trade result is that players reject trade offers that would increase their own material payoff.

In the state game each player is matched with another player in the population, and trade within these two-player subgroups is assumed to be efficiency-enhancing.

\footnotetext{
${ }^{16}$ This set is, again, very large. Suppose, for instance, that the buyer/responder chooses to accept a price offer for a particular $p_{0} \in[0,1]$ but rejects any other price offer, and the seller/proposer offers this price $p_{0}$ if $v_{S} \leq p_{0}$ and demands a price of 1 or higher otherwise. It is straightforward to confirm that these strategies are optimal replies to each other, if chosen simultaneously. Note that the set of Bayesian Nash equilibria includes the PBE and that the PBE is the most efficient equilibrium in this set.
} 
In an evolutionary context where the own material payoff is evaluated in comparison to average material of the rest of the population, the formation of subgroups can often support the emergence of in-group favoritism. In the bargaining game, however, mutation to "more cooperative" behavior within the subgroup is not evolutionarily advantageous; in contrast, evolutionarily stable behavior leads to tough bargaining behavior and the rejection of too low offers. From the outside, this behavior may be interpreted as "revenge" or costly punishment for an offer that is perceived as unfair. But what is behind the rejection behavior is the straightforward evolutionary stability logic developed by Schaffer (1988): Rejecting offers makes a responder relatively better off than trading if trade mainly benefits the proposer and gives the responder much less. The rejection of such offers reduces the responder's payoff, but it reduces the proposer's material payoff by even more. If material payoff relative to that of other players matters, players are more reluctant to accept offers that give them only a small own material payoff, in particular if they expect that acceptance will give the other side a large own material payoff.

The rejection behavior, in turn, affects the evolutionarily stable offer-making. In the role of proposer, players anticipate the responder's reluctance to accept. This has implications for the proposer's choice and tends to make the evolutionarily stable offer more generous. This is true both if the proposer is the informed player and if the proposer is the uninformed player. But in both cases the offer is also shaped by the evolutionary considerations of the proposer. Proposers are reluctant to make concessions and to leave a too high share of the surplus to the responder. Overall, the accommodating behavior of the proposer is not sufficient to compensate for the responder's increased demand. Thus, the evolutionarily stable probability of trade is lower than in the corresponding perfect Bayesian equilibrium, and this effect is strongest in small populations.

\section{A Appendix}

\section{A.1 Proof of Proposition 2}

Equation (4) characterizes the critical level of $v_{S}$ for which the ESS offer $b$ is accepted. As $v_{S}$ is uniformly distributed on $[0,1]$, the probability of trade (6) is obtained directly from inserting $b$ in (2) into (4). This probability is strictly increasing in $m$ with 
$\lim _{m \rightarrow \infty} \omega=1 / 2$. In comparison, in the PBE, the equilibrium offer is $b^{N}=1 / 2$ and this offer is accepted if $v_{S} \leq 1 / 2$ which has a probability equal to $1 / 2$.

The aggregate resource rent that emerges from the take-it-or-leave-it offer in the $\mathrm{PBE}$ is

$$
W^{N}=\operatorname{Pr}\left(v_{S} \leq b^{N}\right) v_{B}+\int_{b^{N}}^{1} v_{S} d v_{S}=\frac{7}{8}
$$

The expected aggregate resource rent for a given pair of players in the equilibrium in ESS is

$$
\begin{aligned}
W & =\left(\frac{1}{2}-\frac{1}{2} \frac{1}{4 m-3}\right) v_{B}+\int_{\frac{1}{2}-\frac{1}{2} \frac{1}{4 m-3}}^{1} v_{S} d v_{S} \\
& =\frac{1}{2}-\frac{1}{2} \frac{(2 m-2)^{2}}{(4 m-3)^{2}}+\frac{2 m-2}{4 m-3} .
\end{aligned}
$$

For $m \rightarrow \infty$ this term converges to $W^{N}=7 / 8$. For smaller $m$ the aggregate rent is smaller. For instance, for $m=1$ we have $W=1 / 2$ : As the probability for trade is zero in this case, the resource stays with the seller/responder who has an expected rent of $1 / 2$. As

$$
\frac{\partial W}{\partial m}=\frac{4 m-2}{(4 m-3)^{3}}>0,
$$

we can also confirm that the aggregate resource rent is monotonically increasing in $m$ in the range $m \in\{1, \ldots \infty\}$.

\section{A.2 Proof of Proposition 3}

We assume that $2 m-1$ players follow the candidate ESS strategy $\left(P_{S}, \pi\right)$ and consider one-step mutations. First we search for the mutant buyer/responder strategy that maximizes the mutant's relative material payoff as defined in (1) for a given ESS behavior of all other players. Then we search for the mutant seller/proposer strategy that maximizes (1), given that all other players use the ESS candidate strategy.

Part 1: buyer/responder strategy mutation: Consider a population with $2 m-1$ ESS players. Their seller/proposer behavior is characterized by a critical value $P_{S}$ such that $p=\pi$ for $v_{S} \leq P_{S}$ and $p=1$ for $v_{S}>P_{S}$. Their buyer/responder strategy is described by a threshold value $\pi$ for the buyer/responder such that the price offer is accepted if and only if $p \leq \pi$. Let there be one mutant who chooses the ESS candidate proposer strategy in the role of seller/proposer, but chooses a possibly 
different threshold price $\pi^{M}$ if the player is in the buyer/responder role. This mutant's expected material payoff at stage 0 is

$$
E_{0} \Pi^{M}=\left\{\begin{array}{ccc}
\frac{1}{2} E \Pi_{S}^{E S S}+\frac{1}{2}\left[P_{S}\left(v_{B}-\pi\right)\right] & \text { if } & \pi^{M}>\pi \\
\frac{1}{2} E \Pi_{S}^{E S S}+0 & \text { if } & \pi^{M}<\pi
\end{array} .\right.
$$

With probability $1 / 2$, the mutant has the role of a seller/proposer, in which he does not behave differently from an ESS player. The term $E \Pi_{S}^{E S S}$ is defined as the expected payoff of an ESS player in the role of seller/proposer if all players behave according to the ESS candidate strategy. This is what the mutant gets in the role of the seller/proposer. With the remaining probability of $1 / 2$, the mutant has the role of a buyer/responder. In this role he chooses $\pi^{M}$, where potentially $\pi^{M} \neq \pi$. The mutant's material payoff in this role depends on the direction in which $\pi^{M}$ deviates. A deviation to a higher acceptance threshold $\pi^{M} \geq \pi$ does not affect the mutant player's own expected material payoff if all other players stick to the ESS candidate strategy (in the role of a buyer he still gets $P_{S}\left(v_{B}-\pi\right)$ where $P_{S}$ is the probability that the seller chooses a price $p=\pi) .{ }^{17}$ Moreover, if $\pi<\pi^{M}<1$, then the material payoff of all other players is also unaffected if they stick to their evolutionarily stable strategies. If $\pi^{M} \geq 1$, then the material payoff of all other players is increased. Thus, deviations to $\pi^{M}>\pi$ weakly reduce the mutant's relative material payoff.

Second, a deviation to a lower acceptance threshold $\pi^{M}<\pi$ causes the breakdown of trade, as the seller proposes a price $p \in\{\pi, 1\}$. This reduces the mutant's expected material payoff by

$$
\frac{1}{2} P_{S}\left(v_{B}-\pi\right)=\frac{1}{2} P_{S}(1-\pi),
$$

compared to a choice of the ESS threshold $\pi$. But it also affects the non-mutant's material payoff if he is in the group with the mutant (which happens to non-mutants with probability $1 /(2 m-1)$ ) and the mutant is assigned the role of the buyer/responder (which happens with probability $1 / 2$ ). Thus, the elimination of trade by a choice of

\footnotetext{
${ }^{17}$ Note that for $\pi^{M} \geq 1$, the mutant's own material payoff in the role of the buyer is equal to $P_{S}\left(v_{B}-\pi\right)+\left(1-P_{S}\right)\left(v_{B}-1\right)=P_{S}\left(v_{B}-\pi\right)$, even though the probability of trade is different in cases $\pi<\pi^{M}<1$ and $\pi^{M} \geq 1$. In the cases in which trade occurs for $\pi^{M} \geq 1$ but not for $\pi<\pi^{M}<1$, this trade occurs at a price $p=1=v_{B}$.
} 
$\pi^{M}<\pi$ reduces the non-mutants' expected material payoff by

$$
\frac{1}{2} \frac{1}{2 m-1} \int_{0}^{P_{S}}\left(\pi-v_{S}\right) d v_{S}=\frac{1}{2} \frac{1}{2 m-1}\left(P_{S} \pi-\frac{\left(P_{S}\right)^{2}}{2}\right) .
$$

Altogether, a deviation to $\pi^{M}<\pi$ eliminates trade and, hence, reduces both the mutant's expected material payoff and that of the average ESS player. Such deviations benefit the mutant type if the expected average loss of ESS players exceeds the expected loss of the mutant, that is, if

$$
\frac{1}{2} \frac{1}{2 m-1}\left(P_{S} \pi-\frac{\left(P_{S}\right)^{2}}{2}\right)>\frac{1}{2} P_{S}(1-\pi) .
$$

This comparison reduces to

$$
P_{S}<2-4 m+4 m \pi
$$

This defines a range $\left(P_{S}, \pi\right)$ in which a deviation from $\pi$ toward $\pi^{M}<\pi$ is a profitable deviation from the ESS candidate strategy. In turn, if

$$
P_{S} \geq 2-4 m+4 m \pi
$$

no profitable deviation from $\pi$ exists and the choice of the ESS threshold $\pi$ yields the maximum relative payoff. In other words, there is a range of $\left(P_{S}, \pi\right)$ for which a mutation that deviates from the ESS candidate buyer/responder strategy $\pi$ is not evolutionarily advantageous. Intuitively, (10) states that the price offer $p=\pi$ cannot be too high.

Part 2: seller/proposer strategy mutation: Consider a population with $2 m-1$ players who behave according to the ESS candidate strategy and one mutant who chooses the ESS candidate value of $\pi$ if the mutant is assigned the role of buyer/responder but who deviates to a different function $P^{M}\left(v_{S}\right)$ in the role of seller/proposer. Analogous to the reasoning in Part 1, price offers $p$ below $\pi$ are all accepted by buyer/responders who follow the ESS candidate strategy. Compared to $p=\pi$, such offers lead to a lower material payoff of the mutant player and a higher average payoff of non-mutant players. Such deviations are evolutionarily disadvantageous. Price offers $p$ above $\pi$ are not accepted by the ESS responders and lead to a breakdown of trade. Thus, the optimal mutant seller/proposer strategy is a threshold strategy with $p=\pi$ for 
$v_{S} \leq P_{S}^{M}$ leading to trade at the largest achievable price for this range $v_{S} \in\left[0, P_{S}^{M}\right]$, and with $p>\pi$ and a breakdown of trade for $v_{S}>P_{S}^{M}$. We search for the optimal threshold $P_{S}^{M}$, which is the own valuation of keeping the good at which the relative benefit of trade at $p=\pi$ equals the relative benefit of no trade.

As the mutant is in the role of a seller/proposer with probability $1 / 2$, the mutant's expected material surplus from trade compared to no trade is

$$
\frac{1}{2} \int_{0}^{P_{S}^{M}}\left(\pi-v_{S}\right) d v_{S}=\frac{1}{2}\left(P_{S}^{M} \pi-\frac{\left(P_{S}^{M}\right)^{2}}{2}\right) .
$$

A mutation in $P_{S}$ also affects the expected payoff of the non-mutant player who is teamed up with the mutant (which happens to a given mutant with probability $1 /(2 m-1))$ in case the mutant is assigned the role of seller/proposer (with probability 1/2). This player's expected material surplus (in the role of the buyer) is equal to $P_{S}^{M}(1-\pi)$. Since all other non-mutant players are not affected by the mutant's choice $P_{S}^{M}$, the mutant's expected additional relative payoff from trade compared to no-trade is

$$
\frac{1}{2}\left(P_{S}^{M} \pi-\frac{\left(P_{S}^{M}\right)^{2}}{2}\right)-\frac{1}{2} \frac{1}{2 m-1} P_{S}^{M}(1-\pi),
$$

which is maximized for

$$
P_{S}^{M}=\frac{2 m}{2 m-1} \pi-\frac{1}{2 m-1} .
$$

Accordingly, a profitable mutation in the role of a seller/proposer does not exist if

$$
P_{S}(\pi)=\frac{2 m}{2 m-1} \pi-\frac{1}{2 m-1}
$$

This defines the ESS choice in the role of the seller/proposer as a function of the buyers' acceptance threshold $\pi$ such that no fitness-increasing deviation exists for mutants in the role of a seller. ${ }^{18}$

The conditions (10) and (11) jointly determine combinations of $P_{S}$ and $\pi$ such that no evolutionarily advantageous deviations exist in the role of the seller or the role of the buyer. Inserting equation (11) for the seller's choice into the "no deviation" condition (10) for the buyer yields an upper bound for $\pi$ (as given in (8)) below which no profitable deviations in the role of the buyer/responder are possible. The

\footnotetext{
${ }^{18}$ Note that $P_{S}(\pi)<0$ if $\pi$ is small. In other words, if $\pi$ is small then trade never occurs: The corresponding ESS choice in the role as seller/proposer is $p\left(v_{S}\right)=1$ for all $v_{S} \in[0,1]$.
} 
combinations $\left(P_{S}, \pi\right)$ define a set of strategies that fulfill the Schaffer (1988) condition for an ESS, and there is a continuum of responder acceptance thresholds $\pi$ and corresponding offer functions $p\left(v_{S}\right)$ that are evolutionarily stable.

\section{References}

[1] Abreu, Dilip, and Rajiv Sethi, 2003, Evolutionary stability in a reputational model of bargaining, Games and Economic Behavior 44(2), 195-216.

[2] Alger, Ingela, and Jörgen W. Weibull, 2013, Homo moralis - preference evolution under incomplete information and assortative matching, Econometrica 81(6), 2269-2302.

[3] Ania, Ana B., 2008, Evolutionary stability and Nash equilibrium in finite populations, with an application to price competition, Journal of Economic Behavior E Organization 65(3-4), 472-488.

[4] Banerjee, Abhijit, and Jörgen W. Weibull, 1993, Evolutionary selection and rational behavior, in: Alan Kirman and Mark Salmon (eds.), Learning and Rationality in Economics, Oxford \& Cambridge (USA), Basil Blackwell, 343-363.

[5] Bar-Gill, Oren, 2006, The evolution and persistence of optimism in litigation, Journal of Law, Economics, and Organization 22(2), 490-507.

[6] Bester, Helmut, and Werner Güth, 1998, Is altruism evolutionarily stable?, Journal of Economic Behavior $\&$ Organization 34(2), 193-209.

[7] Binmore, Ken, Michele Piccione, and Larry Samuelson, 1998, Evolutionary stability in alternating-offers bargaining games, Journal of Economic Theory 80(2), $257-291$.

[8] Chatterjee, Kalyan, and William Samuelson, 1983, Bargaining under incomplete information, Operations Research 31(5), 835-851.

[9] Dekel, Eddie, Jeffrey C. Ely, and Okan Yilankaya, 2007, Evolution of preferences, Review of Economic Studies 74(3), 685-704.

[10] Dufwenberg, Martin, and Werner Güth, 2000, Why do you hate me? - On the survival of spite, Economics Letters 67(2), 147-152. 
[11] Eaton, B. Curtis, Mukesh Eswaran, and Robert J. Oxoby, 2011, 'Us' and 'them': The origin of identity, and its economic implications, Canadian Journal of Economics 44(3), 719-748.

[12] Ellingsen, Tore, 1997, The evolution of bargaining behavior, Quarterly Journal of Economics 112(2), 581-602.

[13] Ely, Jeffrey C., and Okan Yilankaya, 2001, Nash equilibrium and the evolution of preferences, Journal of Economic Theory 97(2), 255-272.

[14] Gale, John, Kenneth G. Binmore, and Larry Samuelson, 1995, Learning to be imperfect: The ultimatum game, Games and Economic Behavior 8(1), 56-90.

[15] Güth, Werner, and Menahem E. Yaari, 1992, Explaining reciprocal behavior in simple strategic games: An evolutionary approach, in: Ulrich Witt (ed.), Explaining Process and Change, Approaches to Evolutionary Economics, The University of Michigan Press, Ann Arbor, 23-34.

[16] Hehenkamp, Burkhard, Alex Possajennikov, and Tobias Guse, 2010, On the equivalence of Nash and evolutionary equilibrium in finite populations, Journal of Economic Behavior 83 Organization 73(2), 254-258.

[17] Heifetz, Aviad, and Ella Segev, 2004, The evolutionary role of toughness in bargaining, Games and Economic Behavior 49(1), 117-134.

[18] Huck, Steffen, and Jörg Oechssler, 1999, The indirect evolutionary approach to explaining fair allocations, Games and Economic Behavior 28(1), 13-24.

[19] Koçkesen, Levent, Efe A. Ok, and Rajiv Sethi, 2000, The strategic advantage of negatively interdependent preferences, Journal of Economic Theory 92(2), 274-299.

[20] Konrad, Kai A., and Florian Morath, 2012, Evolutionarily stable in-group favoritism and out-group spite in intergroup conflict, Journal of Theoretical Biology $306,61-67$.

[21] Lu, Xiaohua, and R. Preston McAfee, 1996, The evolutionary stability of auctions over bargaining, Games and Economic Behavior 15(2), 228-254. 
[22] Maynard Smith, John, 1974, The theory of games and the evolution of animal conflicts, Journal of Theoretical Biology 47(1), 209-221.

[23] Maynard Smith, John, and George R. Price, 1973, The logic of animal conflict, Nature 246 (5427), 15-18.

[24] Mui, Vai-Lam, 1995, The economics of envy, Journal of Economic Behavior \& Organization 26(3), 311-336.

[25] Myerson, Roger B., and Mark A. Satterthwaite, 1983, Efficient mechanisms for bilateral trading, Journal of Economic Theory 29(2), 265-281.

[26] Nowak, Martin A., Karen M. Page, and Karl Sigmund, 2000, Fairness versus reason in the ultimatum game, Science 289 (5485), 1773-1775.

[27] Nowak, Martin A., Akira Sasaki, Christine Taylor and Drew Fudenberg, 2004, Emergence of cooperation and evolutionary stability in finite populations, Nature 428 (6983), 646-650.

[28] Nowak, Martin A., 2006, Evolutionary Dynamics, Harvard University Press, Cambridge, MA.

[29] Ok, Efe A., and Fernando Vega-Redondo, 2001, On the evolution of individualistic preferences: an incomplete information scenario, Journal of Economic Theory $97(2), 231-254$.

[30] Possajennikov, Alex, 2000, On the evolutionary stability of altruistic and spiteful preferences, Journal of Economic Behavior \& Organization 42(1), 125-129.

[31] Robson, Arthur J, 1990, Efficiency in evolutionary games: Darwin, Nash and the secret handshake, Journal of Theoretical Biology 144(3), 379-396.

[32] Schaffer, Mark E., 1988, Evolutionary stable strategies for a finite population and a variable contest size, Journal of Theoretical Biology 132(4), 469-478.

[33] Sethi, Rajiv, 1996, Evolutionary stability and social norms, Journal of Economic Behavior \& Organization 29(1), 113-140.

[34] Sethi, Rajiv, and E. Somanathan, 2001, Preference evolution and reciprocity, Journal of Economic Theory 97(2), 273-297. 
[35] Taylor, Christine, Drew Fudenberg, Akira Sasaki, and Martin A. Nowak, 2004, Evolutionary game dynamics in finite populations, Bulletin of Mathematical Biology 66(6), 1621-1644. 